\title{
Commentary
}

\section{Motor Myosin V Caught on Video: Foot Stomping in Biology}

M

yosins are a large superfamily of eukaryotic motor proteins originally found in muscle cells (hence "myo," from the Greek "mys") that have in common that they bind actin fibers, hydrolyze ATP, and thus generate the mechanical force to directionally walk on the fibers. As a family, myosins are chiefly responsible for both muscle contraction and the organization of vesicles and organelles within eukaryotic cells. A plethora of recent single molecule studies have obtained real-time accounts of such "walks on the wire," primarily using fluorescence microscopy and optical tweezers, especially of myosin V. This particular myosin is a bipedal walker that moves in the direction of the barbed (or + ) end of an actin filament to transport a vesicle cargo. The single molecule studies so far have unequivocally shown that myosin V moves its two "feet" one at a time ("hand-over-hand" mechanism) by taking $\sim 72 \mathrm{~nm}$ steps (which advances the body and cargo by $\sim 36 \mathrm{~nm}$ per step), and have started to reveal some details of how the steps are taken. Their primary limitation, however, has been that typically only one or two specific sites on the feet (often referred to as "heads") or the legs, labeled either with a fluorophore or a microscopic bead, can be visualized in a fluorescence or optical trapping microscope. This limitation has now resoundingly been lifted by a remarkable piece of work recently published by Toshio Ando and colleagues as an article in Nature in which the authors used a high-speed variant of atomic force microscopy (AFM) to directly and at video rate visualize the dynamic walk of unlabeled myosin $\mathrm{V}$ motors on actin fibers. ${ }^{1}$

AFM uses a sharp probe tip mounted at the end of a flexible cantilever to raster scan over an atomically flat surface and record a contour map of molecules deposited on it. ${ }^{2}$ Single biopolymers are typically imaged in "tapping mode" wherein the tip is gently tapping up and down while scanning to avoid excessive forces on the soft sample. The main obstacle to a more widespread use of AFM in biology has long been the difficulty in capturing dynamic biological processes. In 2001, Ando and colleagues $^{3}$ therefore designed a high-speed AFM based on exceedingly small cantilevers of high resonance frequency (450$650 \mathrm{kHz})$ and small spring constant (150-280 pN/nm) to fur-

Published online in Wiley Online Library

(wileyonlinelibrary.com).

DOI 10.1002/bip.21569

(C) 2010 Wiley Periodicals, Inc. ther reduce tip forces and dramatically accelerate scanning so that consecutive images of a $240 \times 240 \mathrm{~nm}^{2}$ area could be captured in as little as $80 \mathrm{~ms}$. The team now found the perhaps most convincing application for this breakthrough technique yet, by applying it to myosin $\mathrm{V}$ bound to actin, which in turn was immobilized on a lipid bilayer deposited on the mineral mica. ${ }^{1}$ The authors directly observed the two myosin legs to "swing" forward in hand-over-hand motion during stochastic bursts of action, and "saw" several up-to-now unobserved features of the walk, including partial unfolding of the motor's coiled-coil tail as evidence for inter-leg strain; "foot stomping," that is, release and reassociation of individual feet, especially at low ATP concentrations; and equilibrium fluctuations between a "bent" and "straight" foot posture in the absence of ATP.

Seeing is believing, and seeing a video of the two conjoined myosin legs taking a stroll on their actin track is material for the textbooks. In addition, it allowed the authors to gain some detailed insight into the "power stroke" mechanism of the motor just by watching more closely under varying buffer conditions, without the need to design a new labeled protein variant for each experimental inquiry. Clearly, high-resolution AFM will now become a staple of the single molecule tool kit in biology. ${ }^{2}$ One can easily imagine other research areas that in the future will be impacted by this new "kid on the block:" From DNA replication and repair to RNA folding to protein conformational change and aggregation. In a decade or two from now, each static structure from X-ray crystallography in our biochemistry textbooks may be accompanied by a dynamic video of the same biopolymer in actual biological action. Stomp your feet if you find this an exciting prospect.

NiLS G. WALTER Professor of Chemistry and Director of the Single Molecule Analysis in Real-Time (SMART) Center The University of Michigan Ann Arbor, U.S.A E-mail: nwalter@umich.edu and jrnlprodbip@cadmus.com

\section{REFERENCES}

1. Kodera, N.; Yamamoto, D.; Ishikawa, R.; Ando, T. Nature 2010, $468,72-76$.

2. Walter, N. G.; Huang, C. Y.; Manzo, A. J.; Sobhy, M. A. Nat Methods 2008, 5, 475-489.

3. Ando, T.; Kodera, N.; Takai, E.; Maruyama, D.; Saito, K.; Toda, A. Proc Natl Acad Sci USA 2001, 98, 12468-12472. 